\title{
Propagant phase in reverberant environments
}

\author{
N. H. Fletcher ${ }^{\mathrm{a})}$ and S. Thwaites \\ Division of Applied Physics, CSIRO, Lindfield 2070, Australia
}

(Received 27 January 1995; revised 25 May 1995; accepted 21 August 1995)

\begin{abstract}
The phase of the transfer function between two points in an extended system can be easily measured if it is taken to be the accumulated phase obtained by smoothly raising the measurement frequency from zero to the reference frequency. Lyon et al., in an extended series of papers [most recently J. Acoust. Soc. Am. 95, 286-296 (1994)], have examined the behavior of this accumulated phase in systems of two and three dimensions and have elucidated the concept of a reverberant phase which is independent of the separation between the two measurement points, provided they are far enough apart, but which rises sharply with increasing frequency. In some applications, for example, in nondestructive testing of extended structures, it is important to be able to observe simple wave-propagation behavior and in particular to measure the propagant phase as a function of frequency and position. The conditions under which this is possible are investigated, and are shown to impose constraints on the ratio between the propagation distance and the size of the structure under test, and either the material damping coefficient or the reflection coefficient at the domain boundaries. These results, which represent an extension of those of Lyon et al., are discussed in terms of the distribution of zeros of the transfer function in the complex frequency plane. Many platelike structures of practical interest are found to satisfy these conditions, so that measurement of propagant phase behavior can provide the basis for a useful technique of nondestructive examination. (c) 1996 Acoustical Society of America.
\end{abstract}

PACS numbers: 43.40.At

\section{INTRODUCTION}

In a significant series of papers over the past decade, ${ }^{1-7}$ Lyon et al. have explored the behavior of the phase of the transfer function in extended systems of one, two, and three dimensions. This phase is normally evaluated by effectively sweeping the measuring frequency from zero up to the frequency of interest, and "unwrapping" the jumps $2 \pi$ in the measured phase to give an "accumulated" phase. This phase is not necessarily equal to the phase difference to be expected from consideration of the number of wavelengths of the propagating wave at the frequency of interest that could be accommodated between the two points, which we might term the propagant phase.

Lyon $^{1}$ showed that, while in a one-dimensional system such as acoustic waves in a pipe the accumulated phase of the transfer function between the generator and an arbitrary measuring point is always approximately equal (within $\pi / 2$ ) to the propagant phase between the two points, the accumulated phase difference in a two-dimensional system is usually many times the magnitude of the propagant phase difference. The difference in behavior is bound up with the behavior of waves reflected from the boundaries of the system, thus forming a pattern of standing waves, and was analyzed by Lyon $^{2}$ in terms of the system's normal modes. He was able to identify the behavior of the accumulated phase as it rose from zero (to within $\pm \pi$ ) for very small separations between excitation and measurement points, to a steady value very much larger than the expected propagant phase at larger

\footnotetext{
${ }^{a)}$ Present address: Research School of Physical Sciences and Engineering, Australian National University, Canberra 0200, Australia.
}

separations. He termed this plateau value the reverberant phase.

Much of the motivation behind this investigation was related to the possibility of recovering clean signals in a reverberant environment by means of inverse filtering. Our present interest is rather different, and concerns the use of propagant phase as a diagnostic tool in nondestructive testing. ${ }^{8,9}$ In either case, however, it is important to be able to distinguish the influence of the reverberant environment on the measured signal. More specifically we explore here the conditions under which it is possible to recover the propagant phase of a signal from measurements made in an extended reverberant environment. It is clear from general considerations that such propagant phase is certainly observed close to the source in systems that are sufficiently large and have sufficient material damping. We shall be concerned therefore with the transition from reverberant to propagant behavior as system size and damping are varied.

\section{POLES AND ZEROS}

The original analysis of Lyon is based upon the analytic form of the transfer function expressed in terms of the normal modes $\psi_{n}$ of the system, where the index $n$ has the dimensionality of the system itself, being $(n, m)$, for example, for the vibration of a planar object. If the time variation is represented by $\exp (j \omega t)$, the mode $n$ has a resonance at angular frequency $\omega_{n}$, at which the damping coefficient is $\alpha$. For simplicity we initially take $\alpha$ to be the same for all modes, corresponding to resonances of constant width. The transfer function between points $\mathbf{r}_{1}$ and $\mathbf{r}_{2}$ at frequency $\omega$ can then be written 


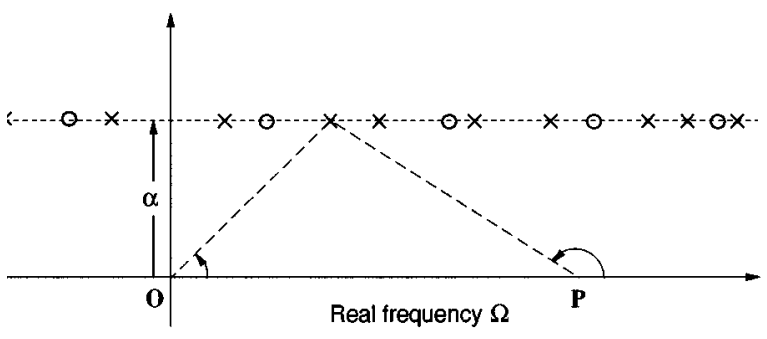

FIG. 1. Poles $\times$ and zeros $\bigcirc$ of the transfer function in the complex frequency plane according to the simple model of Lyon $^{1}$ for a system with frequency-independent damping $\alpha$. The accumulated phase up to real frequency $\Omega$ is evaluated by counting the number of poles and zeros passed or, more properly, by summing the changes in phase angles shown.

$$
Z_{12}(\omega)=\operatorname{const} \times \sum_{n} \frac{\psi_{n}\left(\mathbf{r}_{1}\right) \psi_{n}\left(\mathbf{r}_{2}\right)}{\omega_{n}^{2}-\omega^{2}+2 j \omega \alpha} .
$$

We note that the mode functions $\psi_{n}$ are real, and that the constant depends upon the physical quantities being measured.

It is straightforward to convert the expression (1) to a form that exhibits explicitly the poles of the transfer function in the complex frequency plane. If we write the denominators of the expansion (1) in the form

$$
\omega_{n}^{2}-\omega^{2}+2 j \omega \alpha=\Omega_{n}^{2}-(\omega-j \alpha)^{2},
$$

where

$$
\Omega_{n}=\left(\omega_{n}^{2}-\alpha^{2}\right)^{1 / 2},
$$

then we can rewrite (1) as

$$
Z_{12}(\omega)=\sum_{n}\left[\frac{a_{n}\left(\mathbf{r}_{1}, \mathbf{r}_{2}\right)}{\omega-\Omega_{n}-j \alpha}-\frac{a_{n}\left(\mathbf{r}_{1}, \mathbf{r}_{2}\right)}{\omega+\Omega_{n}-j \alpha}\right],
$$

where the residues $a_{n}\left(\mathbf{r}_{1}, \mathbf{r}_{2}\right)$ are given by

$$
a_{n}\left(\mathbf{r}_{1}, \mathbf{r}_{2}\right)=\mathrm{const} \times \frac{\psi_{n}\left(\mathbf{r}_{1}\right) \psi_{n}\left(\mathbf{r}_{2}\right)}{2 \Omega_{n}} .
$$

The poles are seen to lie at the points $\pm \Omega_{n}+j \alpha$ and thus along a line in the upper half-plane, as shown by the $\times$ symbols in Fig. 1.

Location of the zeros of the transfer function is more difficult, and it was not until the third paper of the series ${ }^{3}$ that this was addressed properly by Tohyama and Lyon. The original analysis of Lyon ${ }^{1,2}$ considered only zeros lying on the line $\omega=\Omega+j \alpha$ in complex frequency space, and noted that, since in the region between two poles the function is dominated by the contributions of those poles, we expect to find a zero if the residues are both of the same sign and no zero if they are of opposite sign. Since the residues are essentially random in sign for a system of two or more dimensions provided that the separation $\left|\mathbf{r}_{2}-\mathbf{r}_{1}\right|$ is large, the average number of zeros is about half the number of poles on any reasonably long segment of this line.

Following the original development of Lyon, the process of measuring the accumulated phase of the transfer function is as shown in Fig. 1. The representative point $P$ moves along the real axis from zero to the measurement frequency $\omega$, and the evolution of the phase is computed by adding the contribution of each pole and zero. If the damping is very small, the poles $\omega_{n}$ of the transfer function lie close to the real axis and, if the system is not large, are well separated. The phase $\phi_{12}$ of the transfer function then decreases by $\pi$ whenever $P$ passes a pole of $Z_{12}$, and conversely increases by $\pi$ whenever $P$ passes a zero. To within an additive constant $\pm \pi$, the phase of the transfer function is thus given by

$$
\phi_{12}(\omega) \approx-\pi\left(N_{P}-N_{Z}\right)
$$

where $N_{P}$ is the number of poles and $N_{Z}$ the number of zeros of $Z_{12}$ below the frequency $\omega$.

For a given upper frequency $\omega$, there is a separation $\left|\mathbf{r}_{2}-\mathbf{r}_{1}\right|$, equal to about half a wavelength at this frequency, below which all the residues $a_{n}\left(\mathbf{r}_{1}, \mathbf{r}_{2}\right)$ are of the same sign, so that $\phi_{12}(\omega)$ is zero to within an additive $\pm \pi$. In a system of two or more dimensions, however, the signs of the residues become random with increasing distance, so that $N_{Z} \approx N_{P} / 2$. The accumulated phase then takes on the large and nearly steady value $\phi_{R} \approx-\pi N_{P} / 2$ that is called the reverberant phase. Experiment ${ }^{1}$ shows that this is what happens in practice for reasonably small systems with low damping.

This simple analysis, however, conceals a dilemma. Clearly the phase of $Z_{12}(\omega)$ should really be evaluated by adding the phase change contributed by each pole and each zero rather than by simply counting their number between zero and the measurement frequency $\omega$. We examine this in terms of the distributions $g_{P}(\Omega)$ and $g_{Z}(\Omega)$, of poles and zeros, respectively, along the pole line $\Omega+j \alpha$. Each pole or zero contributes a phase change that depends upon its frequency $\Omega$ and damping $\alpha$ in the form of the difference between two inverse tangent functions evaluated at the ends of the integration interval $(0, \omega)$ along the real frequency axis. Because $\tan ^{-1} x$ is defined to have a value between $-\pi / 2$ and $\pi / 2$, the discontinuity when $\Omega=\omega$ and $x$ goes through infinity provides the simple count $-\pi\left(N_{P}-N_{Z}\right)$ of (6), and there is a remainder $D(\omega)$ of the form

$$
\begin{aligned}
D= & F(\omega, \alpha, g)-\int_{-\infty}^{\infty}\left[g_{P}(\Omega)-g_{Z}(\Omega)\right]\left[\tan ^{-1}\left(\frac{\alpha}{\Omega-\omega}\right)\right. \\
& \left.-\tan ^{-1}\left(\frac{\alpha}{\Omega}\right)\right] d \Omega \\
\approx & -2 \int_{\omega}^{\infty}\left[g_{P}(\Omega)-g_{Z}(\Omega)\right] \frac{\alpha \omega}{\Omega^{2}} d \Omega
\end{aligned}
$$

where $F(\omega, \alpha, g)$ is a finite constant, the value of which depends upon its arguments. Under the reverberant-phase approximation, $g_{Z} \approx g_{P} / 2$ and the integral in (7) diverges, except in the case of ideally thin rods $\left(g_{P} \sim \Omega^{-1 / 2}\right)$, ideally flexible strings $\left(g_{P} \sim\right.$ constant $)$, or ideally thin plates ( $g_{P} \sim$ constant $)$. For all three-dimensional systems, and thus for all real physical systems, the integral diverges. If the integral diverges, then the remainder $D$ is infinite, and so also is the correction to the expression (6). 


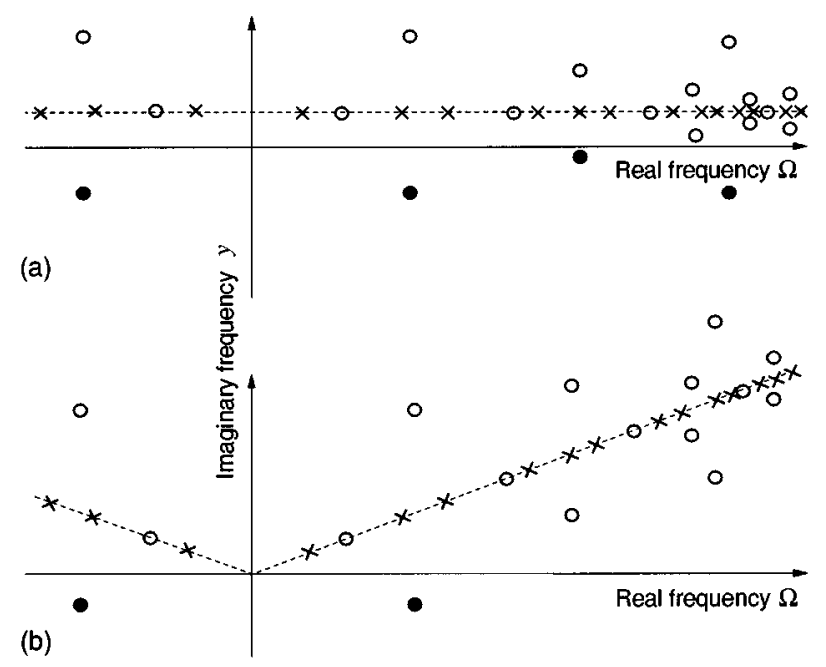

FIG. 2. More realistic distribution of the poles $\times$ and zeros $\bigcirc$ for systems with (a) constant damping and (b) damping increasing with increasing frequency. Zeros are either on the pole line or else distributed in pairs above and below it. Zeros marked with filled circles that lie below the real axis are nonminimum-phase (NMP) zeros and contribute to the reverberant phase.

Although this dilemma escaped attention, its solution appears naturally from later work by Tohyama and Lyon, as we now outline. Clearly, the transfer function given in (4) can be rewritten as

$$
Z_{12}(\omega)=\frac{f(\omega-j \alpha)}{\prod_{n}\left(\omega \pm \Omega_{n}-j \alpha\right)}
$$

and, if we truncate the sum in (7) and thus the product in (8) at some arbitrarily large value $N$ of $n$, then the numerator $f$ is a polynomial of degree $2 N-1$ in $\omega$ while the denominator is a polynomial of degree $2 N$. This conclusion remains valid as $N \rightarrow \infty$. Thus, although the number of zeros on the line $\omega=j \alpha$ is only half the number of poles once the separation and frequency are large enough for the field to be reverberant, the remaining $N$ zeros occur as pairs that are symmetrically placed above and below that line as shown in Fig. 2. Zeros that are above the real axis cancel the phase contribution of an equal number of poles, and it is only the nonminimum-phase zeros (NMP zeros for short) lying below the real axis with which we must be concerned. Convergence of the remainder expression (7) requires that the density of these zeros along the frequency axis must increase less rapidly than $\Omega^{1-\epsilon}$ where $\epsilon$ is a small positive quantity.

We note that the $2 N-1$ zeros do not necessarily all lie within the frequency range $\left(-\Omega_{N}, \Omega_{N}\right)$ on the real frequency axis, though most of them do. In fact we shall see later that, in general, the distribution of zeros is slightly stretched along the frequency axis, relative to the distribution of poles. An examination of the case of two isolated poles with real residues of opposite sign shows that the zero lies outside the frequency interval spanned by the poles. This does not, of course, prove the more general assertion, but suggests its plausibility. We shall see the importance of this point presently. It was mentioned in passing by Tohyama and Lyon, ${ }^{3}$ but its consequences were not investigated.
Consider now the case of a system with constant damping $\alpha$ as the size of the system is increased. Suppose we concentrate attention on a frequency range from zero to some value $\Omega_{\max }$, within which range the numbers of poles and zeros are necessarily finite. As discussed above, the zeros of the transfer function (4) are either on the line $\omega=\Omega+j \alpha$ or distributed in pairs at points $\left(\Omega_{Z, n}+j \alpha\right) \pm j \delta_{n}$, where the quantities $\delta_{n}$ are finite and positive. As the size of the system is increased, keeping its shape constant, modes in the frequency range $\left(0, \Omega_{\max }\right)$ will be compressed into the range $\left(0, \Omega_{\max } / m^{M}\right)$, where $m$ is the linear magnification factor of system size and $M$ is the dimensionality of the system. At the same time, the relative positions of all the poles and zeros about the line $\omega=j \alpha$ will remain geometrically similar to those of the original range, so that the distances $j \delta_{n}$ in the complex plane will become $j \delta_{n} / m^{M}$. The cloud of zeros thus tends to condense toward the pole line as the size of the system is increased. We are concerned, however, not with the general distribution of these zeros, but rather with the absolute number of zeros lying in the lower half-plane.

Tohyama and Lyon ${ }^{3,5}$ gave attention to the distribution of the zeros of $Z_{12}$ and showed that, in the complex plane $\omega=\Omega+j y$, the probability distribution of these zeros behaves asymptotically like $1 / \beta^{2}$, where $\beta=g_{P}(y-\alpha)$ is the normalized distance away from the pole line $\omega=j \alpha$. Because this distribution diverges as $y \rightarrow 0$, they assumed an integrable probability distribution function of the form

$$
p(\beta)=\frac{\sqrt{\epsilon} / \pi}{1+\epsilon \beta^{2}},
$$

where $\epsilon$ is an arbitrary shape constant. [There is a difference of a factor 2 between (9) and the original because we have used signed $\beta$ rather than $|\beta|$.] Since, by their arguments, the density of zeros on the pole line is $g_{P} / 2$, this leaves a density $g_{P} / 2$ of zeros to be described by the probability distribution (9), and the density of NMP zeros becomes

$$
g_{\mathrm{NMP}}=\frac{N_{P}}{2} \int_{-\infty}^{-g \alpha} p(\beta) d \beta .
$$

If the modal density is sufficiently large that $\epsilon \beta^{2} \gg 1$ below the real axis, then we can use the asymptotic form of (9) in (10) and we find that $g_{\mathrm{NMP}}=1 /(2 \pi \alpha \sqrt{\epsilon})$.

If the damping $\alpha$ is constant, then the density of NMP zeros is constant in frequency, while if $\alpha$ increases with increasing frequency, then $g_{\mathrm{NMP}}(\Omega)$ decreases with increasing $\Omega$. It is this density $g_{\mathrm{NMP}}$ that we must use, rather than $g_{Z}-g_{P}$, in the remainder term $D$ of (7). Its form guarantees convergence of this remainder except for the physically unrealistic cases in which the damping is zero or in which the damping actually decreases with increasing frequency at a rate more rapid than $\Omega^{-1}$.

The distribution of poles and zeros, for the case of constant damping, is thus qualitatively of the form shown in Fig. 2(a). As discussed by Tohyama et al., ${ }^{5-7}$ the essentially constant distribution in frequency of NMP zeros below the real axis leads to a steady increase in the phase of the transfer function with frequency, and the derivative of this increase 
can be interpreted as a "group delay" $\tau_{G}$. Since each NMP zero, along with its noncancelled pole, contributes $2 \pi$ to the phase, we can write

$$
\tau_{G}=2 \pi g_{\mathrm{NMP}}=\frac{1}{\alpha \sqrt{\epsilon}} .
$$

If the mode damping $\alpha$ is independent of frequency, then $\tau_{G}$ is also independent of frequency, and we must choose $\epsilon=4$ to give agreement with other studies. ${ }^{5}$ In the more general case in which $\alpha$ increases with increasing frequency, $\tau_{G}$ decreases. When the total modal density, and thus the density of NMP zeros, is small, the increase of phase with frequency may exhibit fluctuations because of the statistical distribution of these zeros. 6,7

Notice that the group delay $\tau_{G}$ does not depend upon the distance between the excitation and measurement points, a characteristic of reverberant behavior that was commented upon by Lyon in the original paper of the series. ${ }^{1}$ We will see later that we need to amend this assertion, and with it the form of the distribution (9), in order to account for propagant behavior.

\section{PROPAGANT PHASE IN AN EXTENDED SYSTEM}

Lyon's demonstration ${ }^{1}$ that one-dimensional systems always exhibit simple propagant-phase behavior rested upon the possibility of expressing the transfer function (1) in simple closed form for the particular case of acoustic waves in a pipe. If the pipe length is $L$ with a source at $x_{1}$ and a detector at $x_{2}$, then

$$
Z_{12}=\operatorname{const} \times \frac{\cos k x_{1} \cos k\left(L-x_{2}\right)}{\sin k L},
$$

where $k=\omega / c$ and we can write $\omega=\Omega+j \alpha$ to allow for the effects of damping. Lyon showed that this case exhibits simple propagant-phase behavior. Since from (12) all zeros lie on the pole line, there are no NMP zeros to be considered, and it is then necessary to ask, in terms of the distribution of poles and zeros, whence the phase shift associated with propagation arises.

The existence of simple propagant phase behavior in this case implies that the number of zeros in any finite frequency range is systematically less than the number of poles by an amount just sufficient to generate the propagant phase. This can be readily established from the form of (12). The number of zeros of the denominator below a particular value of frequency, and thus of $k$, large enough to encompass many resonances is $n_{P} \approx k L / \pi$, while the number of zeros of the numerator $n_{Z} \approx k\left(L+x_{1}-x_{2}\right) / \pi$, both approximations holding to within an additive \pm 1 . The deficit of zeros in the range is thus $n_{P}-n_{Z}=k\left(x_{1}-x_{2}\right) / \pi$ which provides the propagant phase. We must presume that this stretching of the distribution of zeros along the frequency axis is a common feature of all systems, even when we are unable to write down an explicit closed form for the transfer function. This deficit of zeros causes no analytical difficulties because, for any real three-dimensional system, the density of both poles and zeros tends to infinity as the frequency increases.
While this approach can be extended to other onedimensional systems such as thin rods, it is not generally possible to find a closed analytic form for the transfer function for systems of higher dimensionality unless the symmetry of the boundary is high and either $\mathbf{r}_{1}$ or $\mathbf{r}_{2}$ is a point of special symmetry. Nevertheless we know from experiment that it is possible to observe simple wave propagation behavior in two- and three-dimensional domains provided that either the walls or the propagating medium itself are sufficiently absorbent. Observation of such propagant behavior is most clear when the domain is very large compared with the wavelength involved.

We also know, however, that particular wall geometries and positions of the source and measurement points may lead to focused echos that complicate the behavior. This observation suggests that it may not be possible to derive completely general conditions under which propagant behavior can always be observed. The generality of the phenomenon in large enclosures indicates, however, that we should be able to adduce a moderately well founded argument that will apply provided the reverberant field is sufficiently diffuse, and thus for all except such singular cases. We proceed in this spirit.

\section{A. Amplitude conditions}

As a first approach, we note that the measured accumulated phase will be equal to the propagant phase if we can show that the amplitude of the directly propagated wave at the measurement point $\mathbf{r}_{2}$ is always much greater than the amplitude of the reverberant field at that point. Suppose that the source is located at $\mathbf{r}_{1}$ for all our subsequent discussion. Then the point $\mathbf{r}_{1}$ is always a singularity in the wave field, though the nature of the singularity depends upon both the dimensionality of the system and the nature of the differential operator (whether $\nabla^{2}$ or $\nabla^{4}$ ) in the wave equation. At a distance from the singularity that is greater than about half a wavelength, the propagating wave amplitude decreases as

$$
\psi_{P}\left(\mathbf{r}_{2}\right)=\frac{A e^{-\gamma\left|\mathbf{r}_{2}-\mathbf{r}_{1}\right|}}{\left|\mathbf{r}_{2}-\mathbf{r}_{1}\right|^{(M-1) / 2}},
$$

where $M$ is the dimensionality of the domain and $\gamma$ is the spatial attenuation rate for plane-wave propagation. If the boundaries are perfectly reflecting then $\gamma=\alpha / c$ where $c$ is the wave speed and $\alpha$ is the mode damping coefficient introduced in Sec. I. If the energy reflection coefficient $\delta$ is not unity, then this influences the relation between $\alpha$ and $\gamma$, as we show below.

The average amplitude of the reverberant field can be estimated from considerations of energy conservation. Suppose that the mean radius of the domain in $M$ dimensions is $R$ so that its enclosed "volume" is about $V=B R^{M}$ and its surface "area" is $S=B M R^{M-1}$ where $B$ is a number about equal to 3 . The rate at which energy is being supplied to the reverberant field is proportional to

$$
\left[\psi_{P}(R)\right]^{2} c S \delta \approx A^{2} c M B e^{-2 \gamma R} \delta,
$$

where $\delta$ is the energy reflection coefficient of the boundary. The rate at which energy is being lost from the reverberant 
field, taking account of both volume and surface losses, is similarly proportional to

$$
\begin{aligned}
2 \gamma \psi_{R}^{2} V c+\psi_{R}^{2}(1-\delta) S c \approx & B \psi_{R}^{2} R^{M-1} c[2 \gamma R \\
& +(1-\delta) M],
\end{aligned}
$$

where $\psi_{R}$ is the peak amplitude of the reverberant field. Equating the two quantities (14) and (15), we find

$$
\psi_{R}^{2} \approx\left[\frac{\delta M e^{-2 \gamma R}}{R^{M-1}[2 \gamma R+(1-\delta) M]}\right] A^{2} .
$$

The condition that the propagating wave dominate completely over the reverberant field at distance $\left|\mathbf{r}_{2}-\mathbf{r}_{1}\right|=r$ from the source is therefore that

$$
\frac{e^{-2 \gamma r}}{r^{M-1}} \gg \frac{\delta e^{-2 \gamma R}}{R^{M-1}[2 \gamma R+(1-\delta) M]} .
$$

The condition (17) can be satisfied if $r \ll R$, and is facilitated if $\delta \ll 1$ so that the boundaries are highly absorbent, or if $\gamma R \gg 1$ and $r \ll R$, so that the propagating wave is largely absorbed before reaching the boundaries but not before it reaches the observation point.

From (15), which can also be written $2 \alpha B \psi_{R}^{2}$, we can express the modal decay constant $\alpha$ in terms of $\gamma$ and $\delta$ by the relation

$$
\alpha=(c / 2 R)[2 \gamma R+(1-\delta) M]
$$

using which, (17) can be written

$$
\alpha \gg \delta e^{-2 \gamma(R-r)}\left(\frac{r}{R}\right)^{M-1} \frac{c}{2 R} .
$$

If we wish to observe propagant behavior over essentially all of the domain, then we let $r \rightarrow R$ and assume that $\delta \approx 1$, which leads to the condition

$$
\alpha \gg c / 2 R .
$$

We should again make the point that this condition is valid only if the reverberant field is truly diffuse, with no "catastrophe" surfaces or points caused by boundary reflection. In this context, a catastrophe point is one at which the energy density is particularly high for purely geometrical reasons. Such catastrophes are common in both acoustic and optic domains and are described as focused echos or, more generally, as caustic surfaces. Elliptical or ellipsoidal boundaries to the domain can lead to ideal focusing and a maximal divergence from the simple behavior leading to the condition (19), while circular or spherical surfaces lead to somewhat more complex catastrophe surfaces. Our generalized arguments do not refer to the neighborhood of such special points or surfaces.

\section{B. Phase conditions}

Another approach to the problem is by way of our earlier discussion of nonminimum-phase zeros. From that discussion it is clear that the accumulated phase measured at a point at some distance from the source can be considered to have two components. The first is the reverberant component contributed by the NMP zeros and expressed in terms of the group delay $\tau_{G}$ of (11) by $\phi_{R}=\Omega \tau_{G}=\Omega / 2 \alpha$. The second is (a)

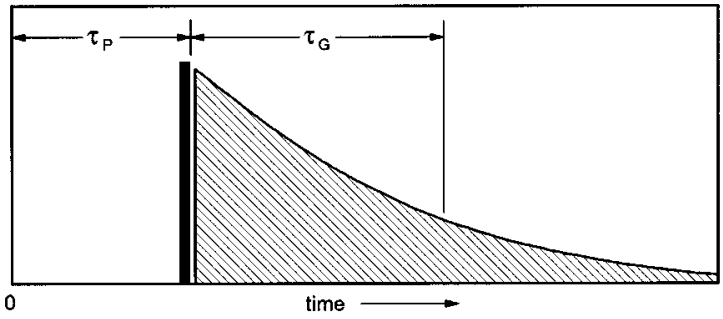

(b)

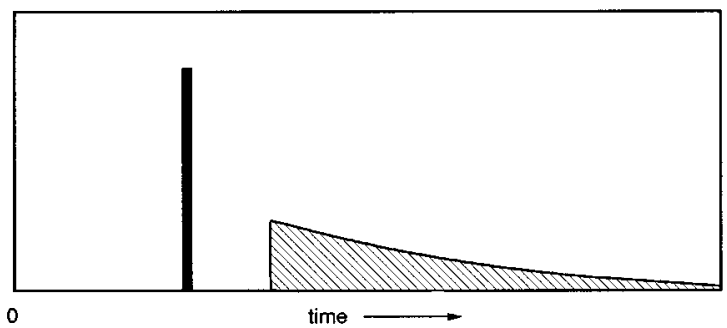

FIG. 3. (a) Schematic version of the signal measured in the time domain following an impulsive excitation at $t=0$. The signal consists of a directly propagated pulse, followed by a diffuse and exponentially decaying reverberant signal. The total time delay is the sum of $\tau_{P}$ and $\tau_{G}$. (b) When the excitation and measurement points are both well distant from the boundary of the domain, the detected signal consists of a directly propagated impulse followed, after a significant time interval, by an attenuated reverberant signal.

the normal propagant phase $\phi_{P}=\Omega r / c$, where $r=\left|\mathbf{r}_{2}-\mathbf{r}_{1}\right|$ is the distance between source and detector. Since these contributions are additive, the condition that propagant phase behavior be observed would appear to be that $\phi_{R} \ll \phi_{P}$ or

$$
\alpha \gg c / 2 r .
$$

We see in the next section, however, that Eq. (21) presents problems.

An interpretation of (21) in the time domain is particularly instructive. For a measurement of this type, as used for example by Tohyama et al., ${ }^{7}$ the source emits a deltafunction impulse and the response measured at a distant microphone is subject to Fourier analysis to recover the phase behavior. Figure 3(a) shows the sort of time record expected in this case. This record can be interpreted in terms of two delays, as we have indicated above. The delay to the first impulse received at the microphone is the phase delay $\tau_{P}$, while the weighted average of the subsequent reverberant signal provides the group delay $\tau_{G}$ which, for an exponentially decaying reverberation, is just $1 / 2 \alpha$. The total weighted delay is effectively $\tau_{P}+\tau_{G}$, and the condition that propagant behavior be observed is then, as discussed above, simply that $\tau_{P} \gg \tau_{G}$ which leads to the condition (21).

\section{Discussion}

It is immediately clear that the conditions (20) and (21) appear to be essentially identical if we let $r \rightarrow R$ in (21). This apparent agreement is, however, spurious. We can see this by comparing the more detailed expressions (19) and (21) for the physically realistic case in which $r<R$ so that measurements are being made relatively close to the source, rather than near the boundaries of the domain. Condition (19) implies that, as is to be expected physically, propagant behavior is most easily observed if the detector is close to the source 
so that the propagant wave amplitude is much greater than that of the reverberant field. The condition (21), however, implies just the opposite behavior-that it becomes increasingly difficult to observe propagant behavior in the close vicinity of the source, in contradiction to physical expectations.

It is possible to modify the time-domain argument adduced to clarify (21) so as to bring these two results into agreement. Suppose that the measurement is being made near the center of a large domain of dimensionality $M$ and that the measurement separation $r$ is very much less than the length $R$ characterizing the size of the domain. The signal received at the detector then has the form shown in Fig. 3(b). There are two significant differences from Fig. 3(a). The first is that there is a time interval between detection of the propagant impulse and the beginning of the reverberant signal. The second is that the amplitude of the reverberant signal is now small relative to that of the propagant signal. If we suppose that reflection from the boundaries of the domain is specular, then the beginning of the reverberant signal consists of a pulse received from an image of the original source that is at a distance of order $R$. The power in this reverberant signal is therefore attenuated by a factor $(r / R)^{M-1}$ relative to the propagant signal. If the absorption coefficient $\gamma$ of the medium is appreciable, then there is a further attenuation by a relative amount $e^{-2 \gamma(R-r)}$. This argument can be extended to include the effects of diffuse reflection at the boundaries and boundary absorption without significant change.

When now we come to determine the energy-weighted average delay time, it is no longer $\tau_{P}+\tau_{G}$ but rather

$$
\bar{\tau} \approx \tau_{P}+(r / R)^{M-1} e^{-\gamma(R-r)} \tau_{G} .
$$

If we require that $\bar{\tau} \approx \tau_{P}$ so that propagant behavior is observed, the result is essentially identical to (19), so that the anomaly is removed.

One might ask why this problem did not appear in the careful experiments of Tohyama et al. ${ }^{7}$ The reason appears to be that the experiments were designed to investigate something rather different. Their signal-processing approach therefore involved, as a first step, "eliminating the pure delay part" from the beginning of the data record. Since this step involved removal of the directly propagated impulse as well as the leading zeros, ${ }^{10}$ we should expect to see only the reverberant signal and thus the group delay $\tau_{G}$.

This argument, however, does not explain why the initial approach involving zero counting gives the result (21) rather than (20), and resolution of this discrepancy is vital to the whole fabric of the theory. We recall that the result (21), when derived in terms of poles and zeros of the transfer function, depends crucially upon the distribution of zeros in the complex plane, and particularly upon the distribution of NMP zeros as given by (9). It is this aspect of the theory that we should therefore reexamine.

The eigenfunctions $\psi_{n}(\mathbf{r})$ that describe the problem, as in (1), are determined in form by the shape of the domain and cannot generally be written down analytically. We can, however, always reexpress the transfer function $Z_{12}(\omega)$ of (1), which in other contexts is referred to as a Green's function, in the form of a spherically symmetrical function
$P\left(\left|\mathbf{r}_{2}-\mathbf{r}_{1}\right|, \omega\right)$ with a spatial singularity at the point $\mathbf{r}_{1}=\mathbf{r}_{2}$, together with a spatially smooth function $Q\left(\mathbf{r}_{1}, \mathbf{r}_{2}, \omega\right)$ contributed by the boundaries. ${ }^{11}$ For simple geometries, $Q$ can be expressed in terms of image sources located outside the problem domain. We then have

$$
Z_{12}\left(\mathbf{r}_{1}, \mathbf{r}_{2}, \omega\right)=P(r, \omega)+Q\left(\mathbf{r}_{1}, \mathbf{r}_{2}, \omega\right),
$$

where $r=\left|\mathbf{r}_{2}-\mathbf{r}_{1}\right|$. All the poles of $Z_{12}(\omega)$ in the complex plane $\omega=\Omega+j y$ are contained in $Q$, and both the positions of the poles and the residues at them are unaltered. A little consideration shows that $P$ is just the infinite-domain propagant function $\psi_{P}$ of (13) and the smooth part $Q$ the reverberant function $\psi_{R}$ of (16).

If we take $k=\Omega / c$ to be the real propagation number for the wave, then $P(r, \omega)$ has an implied factor $e^{-j k r}$ which means that the phase of $P$ depends upon the real part $\Omega$ of $\omega=\Omega+j y$, but $P$ is independent of the imaginary part $y$ in both phase and amplitude. The magnitude of $Q$, on the other hand, derives entirely from the poles of $Z_{12}$ and so decreases steadily with distance away from the line $\omega=j \alpha$. This means that, if $|P|>|Q|$ on the real axis below the measurement frequency $\Omega$, then $|P|>|Q|$ everywhere in the lower halfplane and there can be no zeros of $Z_{12}=P+Q$ below the real axis. What has happened in this circumstance is that the background contributed by the propagant wave has shifted all the NMP zeros of $Z_{12}$ into the upper half-plane, so that no NMP zeros remain.

Because of the statistical nature of the reverberant field, the magnitude of $Q$ on the real axis fluctuates about its mean value $\overline{|Q|}$ in any small frequency range. The condition for observing propagant behavior is therefore that $\overline{|P|} \gg \overline{|Q|}$, with the inequality interpreted as meaning about a factor 2 or 3. From (13) and (16) this condition can be written

$$
\alpha>\delta e^{-2 \gamma(R-r)}\left(\frac{r}{R}\right)^{M-1} \frac{c}{2 R},
$$

which is identical with (19). If this inequality is only just satisfied, then some of the former NMP zeros will have been moved to only just above the real axis and a few may remain in the lower half-plane, so that we expect the phase behavior to be somewhat erratic. This effect will be more pronounced if the reverberant field is not diffuse but suffers from focused echos or other irregularities.

If we set $\delta=1$ and assume $\gamma R \ll 1$, then the right-hand side of (24) is simplified and increased in magnitude, so that the condition so derived,

$$
\alpha \gg\left(\frac{r}{R}\right)^{M-1} \frac{c}{2 R},
$$

should ensure propagant behavior unless the reverberant field is very far from being diffuse.

\section{Distribution of zeros}

The discussion in the previous section shows that, if the propagation distance $r$ is small, then $|P|>|Q|$ except close to the pole line, so that the cloud of zeros must collapse toward that line. Further, since the zeros were initially distributed in pairs on either side of the pole line, and $P$ does 
not depend upon the imaginary component of the frequency, this collapse must be symmetrical. It is important to see how this collapse can be incorporated into the original discussion of the statistics of the distribution of zeros put forward by Tohyama and Lyon.

The reason that the criterion (25) differs from that expressed in (21) is presumably that there are subtle correlations between the signs and magnitudes of the residues at the poles of $Z_{12}$ in the simple expression (1) when the ratio of the propagation distance $r$ to the domain radius $R$ is sufficiently small. While it is difficult to examine this problem from first principles, we can at least see what modification needs to be made to the arguments adduced by Tohyama and Lyon $^{3,5}$ to derive the distribution function (9) in order to retrieve the situation. Such an ad hoc modification does not violate any established features of the statistical theory, since we recall that only the asymptotic form of (9) has a theoretical justification.

There are two possible consequences of the existence of correlations between the signs and magnitudes of the residues of $Z_{12}$ for small $r$. The first, which derives from correlations between the signs, is that the density $g_{Z}^{\mathrm{PL}}$ of zeros lying on the pole line may be greater than the random value of $g_{P} / 2$ for small values of $r$. If this increase has the form

$$
g_{Z}^{\mathrm{PL}}=\frac{1}{2} g_{P}\left\{2-\tanh \left[\mu\left(\frac{r}{R}\right)^{M}\right]\right\},
$$

where $\mu$ is a constant and $M$ is the dimensionality of the domain, then the fraction of zeros lying off the pole line varies as $\tanh \left[\mu(r / R)^{M}\right]$ and we regain very nearly the form of (25) from the argument leading to (21). For a onedimensional system $\mu=0$, since all zeros lie on the pole line, while for systems in two or three dimensions we expect $\mu$ to be of order unity. This suggests that $\mu=(M-1) \mu^{\prime}$, where $\mu^{\prime}$ is another constant, which would take care of this behavior automatically.

A more likely alternative, and one that accords better with our derivation of (25), is that the correlation between the magnitudes of the residues of $Z_{12}$ leads to a functional dependence of the shape parameter $\epsilon$ in (9), of the form

$$
\epsilon=4 \operatorname{coth}^{2}\left[\mu(r / R)^{M}\right] .
$$

This leads to a collapse of the cloud of zeros toward the pole line when $r \ll R$, and to results very similar to (26) for the density of NMP zeros. Such a functional form for $\epsilon$ is not ruled out by the argument leading to (9), since only the asymptotic form of this expression is determined and the introduction of $\epsilon$ as a constant is quite arbitrary. Once again, we expect that $\mu=(M-1) \mu^{\prime}$.

The form of the functional dependences suggested in (26) and (27) should not be taken too seriously. In particular, the hyperbolic tangent function has been introduced simply as a convenient function that tends linearly to zero for small values of its argument and approaches unity asymptotically for large values of the argument. The precise form of the behavior will almost certainly depend upon the shape of the domain and the location of the excitation and observation points within it. There is also the possibility of introducing other nondimensional quantities such as $c / R \Omega$ or $\alpha / \Omega$ into relations, but such a complication does not appear to be necessary.

\section{E. Origin of propagant phase}

While the argument above establishes the conditions under which the contribution of reverberation to accumulated phase is small compared with that of propagation delay, it does not make clear the origin of the propagant phase. Clearly this must arise in some way from details of the distribution of the zeros of the transfer function relative to the poles. We explore two possibilities, one of which can be discarded.

As the size of the system or its damping is increased, the distribution of zeros of the transfer function condenses toward the pole line, this condensation being particularly rapid if the excitation and observation points are separated by a distance that is small compared with the system dimensions, as expressed by (27). The distribution of zeros is, however, a statistical quantity and we can envision the possibility that there is a small density of zeros lying at points $(\Omega \pm j \infty)$ in the complex frequency plane. Despite the condensation, one of these zeros will remain of nonminimum phase, and together the pair and the two poles that they fail to cancel will contribute to the accumulated phase. It is conceivable that the propagant phase arises in this manner, the statistics of these zeros at infinity being determined by the correlation between residues associated with separation between excitation and observation points. This is not the explanation that we favor, if only for the reason that, as discussed in Sec. II above, it does not apply in the case of one-dimensional systems.

The second possibility is that the total distribution of zeros is slightly stretched along the real frequency axis, relative to the distribution of poles, by an amount just sufficient to account for the propagant phase. We showed in Sec. II that this is what happens in one dimension, where we can find an explicit closed expression for the transfer function, and it is natural to expect something similar in two- or threedimensional systems, even though we are unable to demonstrate it in a general case for lack of a closed expression for the transfer function. It is clear, however, that special cases such as a circular membrane excited at its center are one dimensional from a mathematical point of view and so should exhibit zero stretching, and it would be surprising if this did not occur for asymmetric excitation as well. This explanation of the origin of the propagant contribution to the total accumulated phase is therefore preferred, though it must be admitted that it has not been demonstrated unequivocally.

\section{EXPERIMENTAL STUDY}

While it is not the purpose of this paper to report an extended experimental investigation, it is important to verify that our conclusions are borne out by measurements. With this purpose in mind, we have measured the accumulated phase of the transfer function for wave propagation on several plate structures having different characteristics. The measurements were carried out using a Hewlett-Packard 


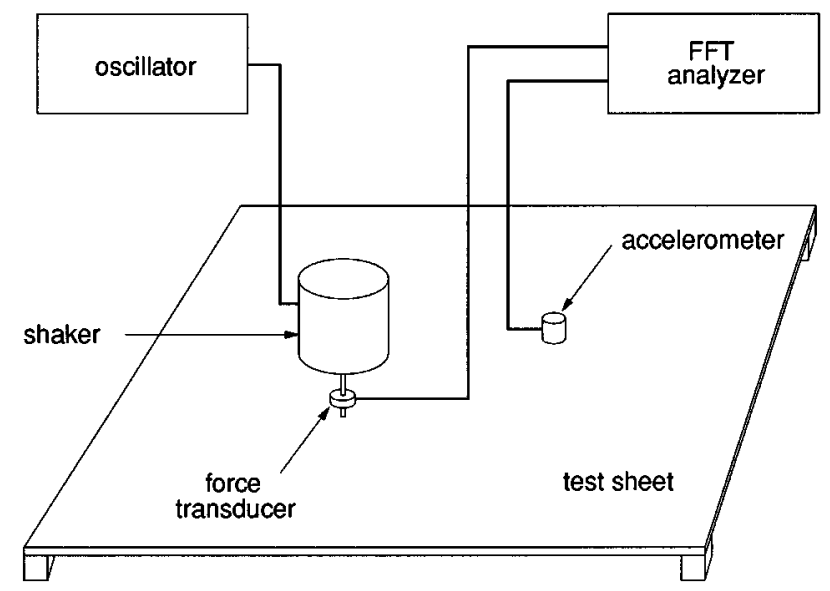

FIG. 4. Measurement setup for the experiments.

model $3567 \mathrm{~A}$ spectrum analyzer and pseudorandom excitation. The reference signal was derived from a force transducer on the exciter head, which was located part way along a diagonal of the plate, and the response signal from a miniature accelerometer, the distance of which from the excitation point could be varied, approximately along the same diagonal. The physical arrangement is shown in Fig. 4, the plate to be measured being supported on blocks at its edges. This system can only approximate the true phase behavior because its resolution becomes less than the mode spacing at high frequencies. It is, however, representative of practical measurement systems.

For each structure, an estimate of the decay constant $\alpha$ was made by observing the decay of an impulsive excitation. Two different methods of giving an adequately sharp impulse were used, and both gave essentially the same results. In the first, the plate was tapped with a thin strip of steel, while in the second a steel ball $8 \mathrm{~mm}$ in diameter was dropped onto the plate from a height of about $15 \mathrm{~cm}$ and caught on the rebound. In each case the decay of the vibration was recorded using a miniature accelerometer connected to a digital oscilloscope. The value of $\alpha$ was taken as the reciprocal of the time required for the amplitude to decay by a factor $e$. Since the shaker does not have zero mechanical impedance, a check was made by repeating the measurement with the shaker attached to the plate. This check confirmed that the influence of the shaker on the decay rate was negligible.

The first structure tested was a uniform aluminum sheet about $900 \mathrm{~mm} \times 700 \mathrm{~mm}$ and $3 \mathrm{~mm}$ in thickness. This sheet had low losses, and there was no absorbing material at its boundaries apart from simple supports. The frequency range covered was such that waves propagated essentially as bending modes throughout, the shortest wavelength being about 6 $\mathrm{cm}$ at a frequency of $6 \mathrm{kHz}$. The measurements of phase behavior at various distances from the excitation point showed rather irregular behavior, as illustrated in Fig. 5. For separations between excitation and measurement points less than about $5 \mathrm{~cm}$, the rate of rise of phase with frequency is comparable with that expected for bending-wave propagation at the separation studied, except for some apparently random jumps of $2 \pi$ which may be due either to isolated

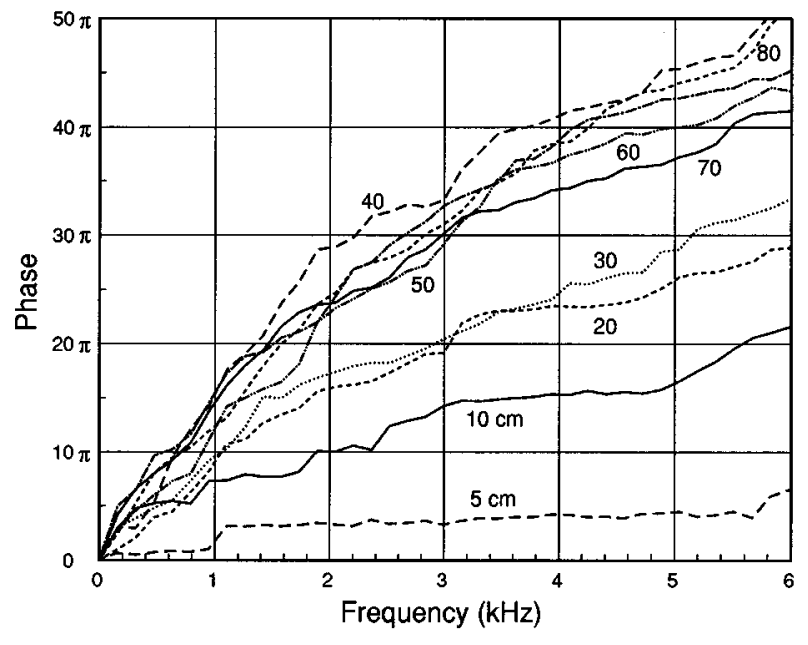

FIG. 5. Accumulated phase as a function of frequency for an aluminum panel, measured at several different separations between source and detector. Note the rapid rise and irregular behavior.

NMP zeros or perhaps to the analysis algorithm. At greater separations the rate of increase of phase is much greater, though this is not a monotonic function of separation for distances in excess of $40 \mathrm{~cm}$. The rate of increase of phase decreases with increasing frequency.

The value of the damping coefficient deduced from reverberation measurements on the sheet is $\alpha \approx 36 \mathrm{~s}^{-1}$ when the signal is low-pass filtered at $2 \mathrm{kHz}$, and $\alpha \approx 56 \mathrm{~s}^{-1}$ when it is high-pass filtered at $10 \mathrm{kHz}$. Setting $M=2$ in (24) and noting that $c \approx 2.2 \omega^{1 / 2} \mathrm{~m} \mathrm{~s}^{-1}$ for this plate, which is about $300 \mathrm{~m} \mathrm{~s}^{-1}$ at $3 \mathrm{kHz}$, we expect to observe propagant behavior only for $r$ less than about $5 \mathrm{~cm}$. This is in good agreement with the results displayed in Fig. 5. The phase curve for $r=5$ $\mathrm{cm}$ shows very nearly propagant phase behavior, except for a few jumps of $2 \pi$ that can be attributed to residual NMP zeros, while the curves for higher separations have high rates of phase increase that can be attributed to reverberant behavior. The difference in slope between the reverberant curves at low and high frequencies is about a factor 2 , which is what we expect from the measured frequency variation of $\alpha$. The rather low modal density associated with bending waves contributes to the irregularity shown in the phase plots in the figure.

An alternative analysis of this data is given in Fig. 6, in which the accumulated phase at a given frequency is plotted as a function of separation between excitation and measurement points. The sharp rise and the reverberant phase plateau, first discussed by Lyon, ${ }^{1}$ are both clear. The fact that the reverberant phase plateau rises less than linearly with frequency in this range is again an expression of the fact that damping increases with frequency. The quantitative agreement between theory and experiment for the reverberant phase plateau values, obtained by integrating $1 / 2 \alpha$ over the frequency range concerned, is not good, however-the calculated reverberant phase is, for each frequency, two to three times the measured phase.

The solution to this apparent anomaly lies in the physical properties of the system. The aluminum sheet is sufficiently thin that wave propagation is by bending waves over 


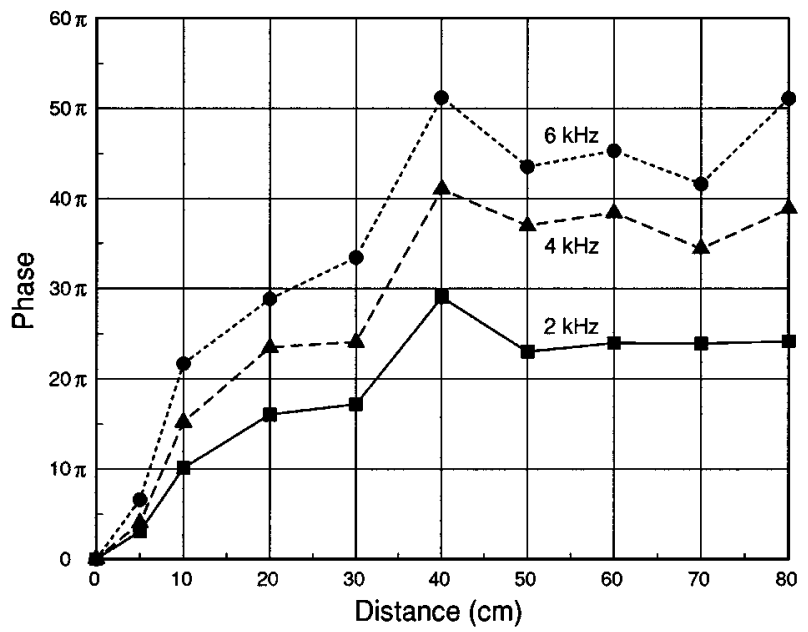

FIG. 6. Accumulated phase as a function of distance between source and detector for an aluminum panel, measured at several frequencies. The plateau is the reverberant phase in each case.

the whole frequency range studied and, as discussed before, the modal density $g_{P}(\Omega)$ for this case is constant, the actual value being $(S / 4 \pi)(\rho h / K)^{1 / 2}$, where $S$ is the plate area, $h$ its thickness, $\rho$ its material density, and $K$ its bending stiffness. The modal overlap, defined by the quantity $H=\alpha g_{P}$, is therefore only about 0.45 so that it is not legitimate to use the asymptotic form $\pi / \sqrt{\epsilon} \beta^{2}$ of the probability distribution of zeros (9). Retaining the full form (9) then leads not to the expression (11) for the group delay $\tau_{G}$ but rather to the smaller quantity

$$
\tau_{G}^{\prime}=\tau_{G} H\left[1-(2 / \pi) \tan ^{-1}(2 H / \pi)\right],
$$

as has been pointed out by Tohyama et al. ${ }^{5}$ For the particular aluminum plate used in our experiment, $g_{P}(\Omega) \approx 0.01$ and $\alpha \approx 50$ over the range of measurement, so that $H \approx 0.5$ and $\tau_{G}^{\prime} \approx 0.4 \tau_{G}$. This leads to a factor 0.4 in the expected reverberant phase plateau and adequately explains our experimental results.

It is informative to discuss the plots of Fig. 6 against the background of our tentative resolution of the conflict between Eqs. (24) and (21) as set out in (25) and (26). For this aluminum sheet, the value of the equivalent radius $R$ on an area basis is $45 \mathrm{~cm}$ and the dimensionality $M=2$. If we assume that $\mu \approx 1.5$ in (26), then we expect the measured phase to rise steadily with distance $r$ up to about $r \approx 40 \mathrm{~cm}$ before saturating at the reverberant phase plateau appropriate to the measurement frequency. This is just what is observed in Fig. 6, lending confirmation to our proposals.

The next structure investigated was a uniform plane honeycomb panel with carbon fibre reinforced composite enclosing sheets, actually part of an aircraft structure. The panel thickness was about $10 \mathrm{~mm}$ and the piece studied was about 1-m square. This panel was thick enough that wave propagation was essentially in the form of shear waves above about $5 \mathrm{kHz}$, but below this frequency the waves had some bending component. The internal damping was very high because of the properties of the honeycomb core. As a result, the phase characteristic showed essentially simple propagant behavior for source and detector located almost anywhere on

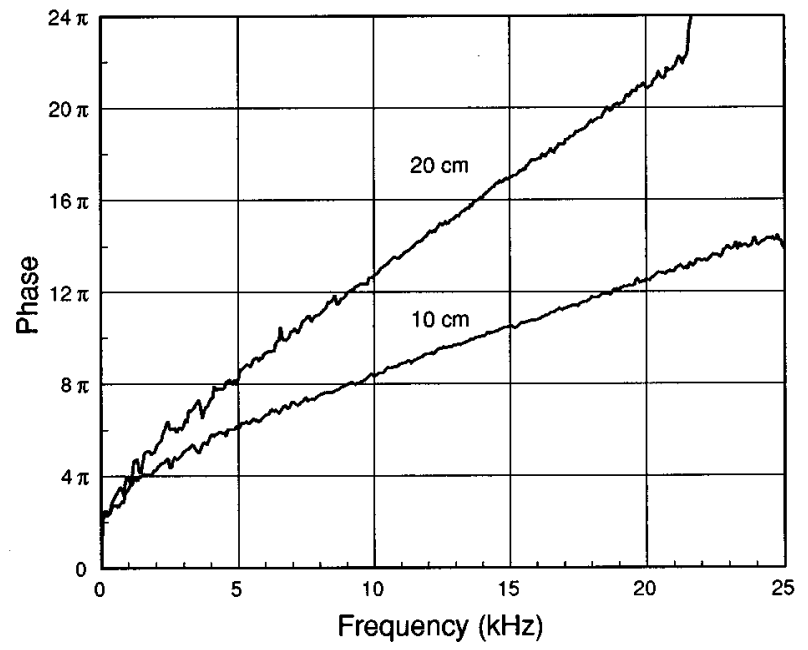

FIG. 7. Accumulated phase as a function of frequency for a composite panel, measured at two different separations between source and detector. The slope of the curve is the propagant phase delay, with the curvature at low frequencies showing bending wave dispersion.

the panel. Close proximity of the measuring point to one of the free edges did not cause any significant change in behavior.

The results of two sets of measurements, for propagation distances of 10 and $20 \mathrm{~cm}$, respectively, are shown in Fig. 7. The simple propagant phase behavior is clearly evident, the only complication being a jump of $2 \pi$ of the upper curve, which may either be instrumental or else reflect the presence of an isolated NMP zero. The slight curvature of the plot at low frequencies indicates the presence of a dispersive bending component in the otherwise predominantly shear waves. It is not clear whether or not there is some contribution from NMP zeros at very low frequencies or whether the apparent offset of $2 \pi$ is instrumental in origin.

From the slope of the phase curves, the wave speed in the plate is about $600 \mathrm{~m} \mathrm{~s}^{-1}$ and $R \approx 0.5 \mathrm{~m}$, so that from (24) the condition that propagant behavior be observed for a propagation distance of $20 \mathrm{~cm}$ is that $\alpha>300 \mathrm{~s}^{-1}$. Measure-

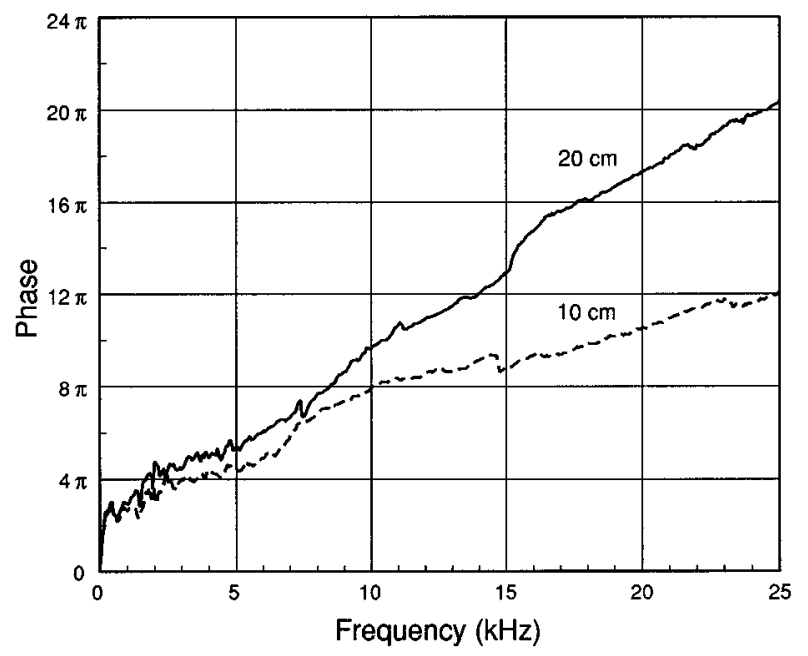

FIG. 8. Accumulated phase as a function of frequency for a table top, measured at two different differences between source and detector. 
ments of the value of $\alpha$ were a little difficult because of the high decay rate of the signal and some anomalies in behavior. The estimated value of $\alpha$ is greater than $500 \mathrm{~s}^{-1}$ and probably close to $2000 \mathrm{~s}^{-1}$, which adequately satisfies this condition and indeed allows propagant behavior to be observed over essentially the whole plate.

Finally, to show the generality of the behavior, we carried out a similar set of measurements on a space about $1 \mathrm{~m}$ across cleared on a wooden laboratory bench of unknown structure and overall dimensions about $2 \mathrm{~m} \times 1.5 \mathrm{~m}$. The audible response of the bench to a sharp blow indicated that it was highly damped, and the measured value of $\alpha \approx 1000 \mathrm{~s}^{-1}$ confirms this. Typical records for this case, at propagation distances of 10 and $20 \mathrm{~cm}$, are shown in Fig. 8. The results show simple nondispersive shear-wave propagation over a wide frequency range, with a degree of irregularity which is probably due to the more complicated structure and properties of the bench.

\section{CONCLUSIONS}

This discussion has clarified the conditions under which reverberant phase or propagant phase behavior may be observed in extended systems. The condition for observation of propagant phase behavior is expressed in Eq. (25), or in greater detail in (24). Our conclusions are not in disagreement with those of Lyon et al., but extend them. Reverberant phase behavior is characteristic of systems with more than one dimension that have low damping, particularly when the measurement point is distant from the source. It is shown particularly well in bending wave propagation on metal panels. Conversely, simple propagant behavior is observed in well-damped systems and particularly when the measurement point is close to the source. These conclusions are by no means surprising, but the detailed examination above has shown how they are related to the modal analysis of these structures.

In the process of reexamination of the theory underlying the phase characteristics of the transfer function, we have been led to extend the conclusions of Lyon et al. concerning the distribution of zeros of the transfer function in the complex frequency plane, as set out in Eq. (26) or (27). This extension, although tentative in form, removes the anomalies that we identified in the original treatment and allows its extension to a wider range of physical situations.

The robustness of propagant phase behavior in platelike structures such as honeycomb composite panels, and even in thick panels of rather lower damping, confirms the basis upon which nondestructive testing procedures involving measurements of propagant phase have been developed.

\section{ACKNOWLEDGMENT}

The authors are greatly indebted to one of the referees who pointed out a serious error in the initial version of this paper. Correction of this error has led to a reformulation and extension of our major conclusions.

${ }^{1}$ R. H. Lyon, "Progressive phase trends in multi-degree-of-freedom systems," J. Acoust. Soc. Am. 73, 1223-1228 (1983).

${ }^{2}$ R. H. Lyon, "Range and frequency dependence of transfer function phase," J. Acoust. Soc. Am. 76, 1433-1437 (1984).

${ }^{3}$ M. Tohyama and R. H. Lyon, "Zeros of a transfer function in a multidegree-of-freedom vibrating system," J. Acoust. Soc. Am. 86, 1854-1863 (1989).

${ }^{4}$ M. Tohyama and R. H. Lyon, "Transfer function phase and truncated impulse response,” J. Acoust. Soc. Am. 86, 2025-2029 (1989).

${ }^{5}$ M. Tohyama, R. H. Lyon, and T. Koike, "Reverberant Phase in a room and zeros in the complex frequency plane," J. Acoust. Soc. Am. 89, 17011707 (1991).

${ }^{6}$ M. Tohyama, R. H. Lyon, and T. Koike, "Statistics on reverberant transfer functions," Second International Congress on Recent Developments in Air and Structure-Borne Sound and Vibration, Auburn University, Alabama, 4-6 March 1992.

${ }^{7}$ M. Tohyama, R. H. Lyon, and T. Koike, "Phase variabilities and zeros in a reverberant transfer function," J. Acoust. Soc. Am. 95, 286-296 (1994).

${ }^{8}$ N. H. Clark and S. Thwaites, "Local phase velocity measurements in plates," J. Sound Vib. (in press).

${ }^{9}$ S. Thwaites and N. H. Clark, "Non-destructive testing of honeycomb sandwich structures using elastic waves," J. Sound Vib. (in press).

${ }^{10} \mathrm{M}$. Tohyama (personal communication, 1994).

${ }^{11}$ P. M. Morse and H. Feshbach, Methods of Theoretical Physics (McGrawHill, New York, 1953), Vol. 1, pp. 808-820. 